\title{
Non-local kinetic energy functional for an arbitrary number of Fermions moving independently in one-dimensional harmonic oscillator potential
}

\author{
N.H. March, P. Senet *, V.E. Van Doren \\ Department of Physics, University of Antwerp (RUCA), Groenenborgerlaan, Antwerp, Belgium
}

Received 1 March 2000; received in revised form 18 April 2000; accepted 18 April 2000

Communicated by B. Fricke

\begin{abstract}
For one-dimensional Fermions bound by a general one-body potential $V(x)$, the Pauli potential is first related to the kinetic energy and the particle density $\rho(x)$. For the model of the harmonic oscillator, $V(x)=\frac{1}{2} x^{2}$, this equation leads to a non-local kinetic energy functional in which only first-order derivatives of $\rho(x)$ enter. This example shows the usefulness of a new concept, the Pauli function, which encompasses the Pauli principle in terms of the electronic density. For the harmonic oscillator model, the kinetic energy can then be expressed exactly in terms of the Thomas-Fermi kinetic energy functional, together with the von Weizsäcker inhomogeneity term, but now in a fully non-local way. (C) 2000 Published by Elsevier Science B.V. All rights reserved.
\end{abstract}

The description of the ground-state of many-electron systems by the ground-state density, the so-called density-functional theory [1], goes back to the pioneering work of Thomas [2] and Fermi [3]. Their theory can be derived from a variational principle (see, for instance, Ref. [4])

$\delta(E-N \mu)=0$,

where $N$ is the number of Fermions, $\mu$ is the chemical potential and $E$ is the energy in terms of the density $\rho(\boldsymbol{r})$.

\footnotetext{
* Corresponding author. Tel.: +32-3-2180 318; fax: +32-32180331.

E-mail address: psenet@ruca.ua.ac.be (P. Senet).
}

Our central aim in this Letter is to focus, for independent Fermions in one-dimension, on the kinetic energy functional $T=T[\rho]$. For the independent Fermions we consider throughout, the energy $E[\rho]$ is then

$E[\rho]=T[\rho]+\int_{-\infty}^{+\infty} d x \rho(x) V(x)$,

where the potential $V(x)$ binds the Fermions such that

$$
\int_{-\infty}^{+\infty} d x \rho(x)=N
$$


In the Thomas-Fermi method the kinetic energy $T_{\mathrm{TF}}$ in one-dimension is given by

$T_{\mathrm{TF}}=c_{k} \int_{-\infty}^{+\infty} d x \rho^{3}(x)$,

where $c_{k}=\frac{\pi^{2}}{24}$, see, for instance, Ref. [5] (atomic units are used throughout). Eq. (4) is useful when $V(x)$ and $\rho(x)$ vary by but a small fraction of themselves over a characteristic de Broglie wavelength for a particle at the Fermi level. When this condition is not satisfied, one must correct Eq. (4) for density gradients and the first proposal goes back to von Weizsäcker [6] (see also [7]) who proposed to add to Eq. (4) in this case the so-called inhomogeneity kinetic energy $T_{\mathrm{w}}$ such that the new kinetic energy $T[\rho]$ was given in this approximation by

$T_{\mathrm{TFW}}=c_{k} \int_{-\infty}^{+\infty} d x \rho^{3}(x)+T_{\mathrm{W}}$,

where

$T_{\mathrm{W}}=\frac{1}{8} \int d x \frac{\left[\frac{\partial \rho(x)}{\partial x}\right]^{2}}{\rho}$.

However, for quantitative work, this kinetic energy functional is not adequate and in this Letter our object is to develop the theory of $T[\rho]$ within a truly non-local framework, for independent Fermions moving in one-dimension.

A central feature of the present work is the socalled differential virial theorem given by March and Young [8]. With the kinetic energy/unit length $t(x)$ defined here from the one-body wave functions $\psi_{i}(x)$ by

$t(x) \equiv-\frac{1}{2} \sum_{i=1}^{N} \psi_{i}^{*}(x) \frac{\partial^{2} \psi_{i}(x)}{\partial x^{2}}$,

their result has the form

$$
\frac{\partial t(x)}{\partial x}=-\frac{1}{2} \rho(x) \frac{\partial V(x)}{\partial x}-\frac{1}{8} \frac{\partial^{3} \rho(x)}{\partial x^{3}},
$$

which holds provided that the boundary conditions are such that $\int d x \nabla^{2} \rho(x)=0$. We next invoke the Euler equation of the density functional theory in the form [1]

$\mu=\frac{\delta T}{\delta \rho(x)}+V(x)$, where $\mu$ was introduced already in Eq. (1) and is constant throughout the entire inhomogeneous Fermionic density distribution $\rho(x)$. Thus, using this constancy of $\mu$, Eq. (8) can be immediately rewritten as

$$
\frac{\partial t(x)}{\partial x}=\frac{1}{2} \rho(x) \frac{\partial}{\partial x}\left[\frac{\delta T}{\delta \rho(x)}\right]-\frac{1}{8} \frac{\partial^{3} \rho(x)}{\partial x^{3}} .
$$

This is the point at which it will be instructive to introduce the Pauli potential $V_{\mathrm{P}}(x)$. The origins of this concept are reviewed by Levy and Görling [9] and the study of Herring and Chopra $[10 ; 11]$ can also be usefully consulted. One definition of the Pauli potential is through the kinetic energy functional $T$ and $T_{\mathrm{W}}$, namely

$V_{\mathrm{P}}(x)=\frac{\delta T}{\delta \rho(x)}-\frac{\delta T_{\mathrm{W}}}{\delta \rho(x)}$.

Hence, by substituting Eq. (11) in Eq. (10), one reaches the result

$$
\begin{aligned}
& \frac{\partial t(x)}{\partial x}-\frac{1}{2} \rho(x) \frac{\partial V_{\mathrm{P}}}{\partial x} \\
& =\frac{1}{2} \rho(x) \frac{\partial}{\partial x}\left[\frac{\delta T_{\mathrm{W}}}{\delta \rho(x)}\right]-\frac{1}{8} \frac{\partial^{3} \rho(x)}{\partial x^{3}} .
\end{aligned}
$$
find

Using the definition of $T_{\mathrm{W}}$ in Eq. (6) one readily

$$
\frac{\delta T_{\mathrm{W}}}{\delta \rho(x)}=\frac{1}{8}\left[\frac{\rho^{\prime}(x)}{\rho(x)}\right]^{2}-\frac{1}{4} \frac{\rho^{\prime \prime}(x)}{\rho(x)},
$$

in which $\rho^{\prime}=\frac{\partial \rho}{\partial x}$, etc. Thus the $x$ derivative of $\delta T_{\mathrm{W}} / \delta \rho(x)$ required in Eq. (12) is easily found and substituting this in Eq. (12) one is led to the exact relation between $t(x), V_{\mathrm{P}}(x)$ and $\rho(x)$ in the form

$$
\begin{aligned}
t^{\prime}(x) & -\frac{1}{2} \rho(x) V_{\mathrm{P}}^{\prime}(x) \\
= & \frac{1}{4}\left[\frac{\rho^{\prime \prime}(x) \rho^{\prime}(x)}{\rho(x)}-\frac{\rho^{\prime 3}(x)}{2 \rho^{2}(x)}+\rho^{\prime \prime \prime}(x)\right] \\
= & \rho^{\prime}\left[\frac{\rho^{\prime \prime \prime}(x)}{4 \rho^{\prime}(x)}-\frac{\delta T_{\mathrm{W}}}{\delta \rho(x)}\right] .
\end{aligned}
$$


Since Eqs. (12) and (14) have a central role in the present study, we stress that the input used in their derivations is the differential virial theorem Eq. (8), the Euler Eq. (9) and the definition of the Pauli potential Eq. (11).

To illustrate the use of Eq. (14) in one-dimensional density functional theory, we shall apply it below to the admittedly specialized model of $N$ independent Fermions filling singly the levels of the linear harmonic oscillator potential $V(x)=\frac{1}{2} x^{2}$. For this example, Lawes and March [12] have derived the following differential equation for the groundstate density $\rho(x)$

$$
-\frac{1}{2} \rho(x) V^{\prime}(x)-\frac{\rho^{\prime \prime \prime}(x)}{8}=(N-V(x)) \rho^{\prime}(x) .
$$

The lowest level only being occupied corresponds to $N=1$ in Eq. (15).

Using again Eq. (8), but now in the example of the harmonic potential model, it follows from Eq. (15) that

$\frac{t^{\prime}(x)}{\rho^{\prime}(x)}=N-V(x)$.

Invoking the Euler Eq. (9) one reaches the result

$$
\left[\frac{t^{\prime}(x)}{\rho^{\prime}(x)}\right]^{\prime}=\left[\frac{\delta T}{\delta \rho(x)}\right]^{\prime} \text {. }
$$

Differentiating Eq. (11) with respect to $x$ and using Eq. (17), we find the desired expression for the derivative of the Pauli potential entering the general Eq. (14), but now for the oscillator model only as

$V_{\mathrm{P}}^{\prime}(x)=\left[\frac{t^{\prime}(x)}{\rho^{\prime}(x)}-\frac{\delta T_{\mathrm{W}}}{\delta \rho(x)}\right]^{\prime}$.

Inserting Eq. (18) into Eq. (12), we find an explicit differential equation for the kinetic energy density (i.e. per unit length) in the one-dimensional harmonic oscillator model for arbitrary number $N$ of independent Fermions as

$t^{\prime}(x)-\frac{1}{2} \rho(x)\left[\frac{t^{\prime}(x)}{\rho^{\prime}(x)}\right]^{\prime}=-\frac{\rho^{\prime \prime \prime}(x)}{8}$.
The object of the rest of this Letter is to solve Eq. (19) to obtain $t[\rho]$, and thus $T[\rho]$, the kinetic energy functional for this one-dimensional harmonic oscillator model. To do so, it is convenient to make the substitution in Eq. (19) that

$Q(x) \equiv \frac{t^{\prime}(x)}{\rho^{\prime}(x)}$,

and hence

$Q^{\prime}(x)-2 \frac{\rho^{\prime}(x)}{\rho(x)} Q(x)=\frac{\rho^{\prime \prime \prime}(x)}{4 \rho(x)}$.

Using an integrating factor, one has

$\rho^{2}(x)\left[\rho^{-2}(x) Q(x)\right]^{\prime}=\frac{\rho^{\prime \prime \prime}(x)}{4 \rho(x)}$,

and hence

$Q(x)=\frac{\rho^{2}(x)}{4} \int^{x} d s \frac{\rho^{\prime \prime \prime}(s)}{\rho^{3}(s)}$.

To determine the constant of integration (actually $N$-dependent) in Eq. (23), we note from Eq. (16) that $Q(0)=N$.

Hence using Eqs. (20) and (23) one finds

$$
\begin{aligned}
t^{\prime}(x)= & N \frac{\rho^{2}(x) \rho^{\prime}(x)}{\rho^{2}(0)} \\
& +\frac{\rho^{2}(x) \rho^{\prime}(x)}{4} \int_{0}^{x} d s \frac{\rho^{\prime \prime \prime}(s)}{\rho^{3}(s)} .
\end{aligned}
$$

Integrating by parts in Eq. (25) and using the boundary condition $t(-\infty)=t(+\infty)=0$ one readily obtains for $-\infty \leq x \leq+\infty$

$$
\begin{aligned}
t(x)= & -\frac{\rho^{\prime \prime}(x)}{12} \\
& +\left[\frac{N}{3 \rho^{2}(0)}+\frac{1}{12} \int_{0}^{x} d s \frac{\rho^{\prime \prime \prime}(s)}{\rho^{3}(s)}\right] \rho^{3}(x) .
\end{aligned}
$$

The third derivative of $\rho$ in Eq. (26) can be reduced to a first derivative of $\rho$ by integrating by parts the integral in the brackets which leads after elementary calculations to the following interesting expression for $t(x)$

$t(x)=t_{w}(x)+\xi_{k}(x) t_{\mathrm{TF}}(x)$, 
in which we have defined the von Weizsäcker kinetic energy density $t_{w}(x)$ and Thomas-Fermi energy density $t_{\mathrm{TF}}(x)$ by respectively,

$t_{w}(x) \equiv \frac{1}{8}\left[\frac{\rho^{\prime}(x)}{\rho(x)}\right]^{2}$,

and

$t_{\mathrm{TF}}(x) \equiv c_{k} \rho^{3}(x)$,

where $c_{k}$ is the same constant as in Eq. (4). The dimensionless function $\xi_{k}(x)$ is given by

$\xi_{k}(x) \equiv \frac{t(0)-t_{w}(0)}{t_{\mathrm{TF}}(0)}+\frac{1}{2 c_{k}} \int_{0}^{x} d s \frac{\left[\rho^{\prime}(s)\right]^{3}}{\rho^{5}(s)}$,

where from Eq. (26)

$t(0)=\frac{N}{3 \rho^{2}(0)}-\frac{\rho^{\prime \prime}(0)}{12}$.

One can easily verify that the function $\xi_{k}(x)$ in Eq. (30) can be reformulated entirely in terms of the kinetic energy densities $t_{w}(x)$ and $t_{\mathrm{TF}}(x)$ :

$\xi_{k}(x)=\xi_{k}(0)+\frac{4}{3} \int_{0}^{x} d x\left[\frac{t_{\mathrm{W}}^{\prime}(x)}{t_{\mathrm{TF}}(x)}-\left[\frac{t_{\mathrm{W}}(x)}{t_{\mathrm{TF}}(x)}\right]^{\prime}\right]$.

The kinetic energy functional can be then deduced formally from Eq. (27) as

$$
\begin{aligned}
T & =\int_{-\infty}^{+\infty} d x t(x) \\
& =T_{\mathrm{W}}+\int_{-\infty}^{+\infty} d x \xi_{k}(x) t_{\mathrm{TF}}(x),
\end{aligned}
$$

in which we emphasize that $\xi_{k}(x)$ is an explicit non-local functional of the electronic density given by Eq. (32).

$T$ is thus the sum of the von Weizsäcker kinetic energy and a term which takes into account the Pauli principle, the so-called Pauli kinetic energy from which derives the Pauli potential used above $[10 ; 11$. The Pauli energy derived here is closely related to the venerable Thomas-Fermi kinetic functional $[2 ; 3]$ through what we term here a Pauli function $\xi_{k}(x)$ which depends nonlocally on the density and its gradient throughout the whole of space. In fact, Eq. (33) also encompasses exactly the limit of a uniform one-dimensional electron gas. Indeed, for such a gas $\rho$ and $t(x)$ are constant and $t$ is equal to $t_{\mathrm{TF}}$ which implies from Eq. (30) that $\xi_{k}(x)=1$ and thus $T=$ $T_{\mathrm{TF}}$ because $T_{\mathrm{W}}=0$. The functional $T$ given by Eq. (33) is therefore an exact relation both for the harmonic oscillator and for the non-interacting uniform electron gas.

The form of the kinetic energy in which a modified TF term enters Eq. (33) has been already anticipated a long time ago [13]. The relations (33) and (27) have been derived by several authors [14-16] within approximate frameworks. For instance, Deb and Ghosh expressed $\xi_{k}(x)$ as a sum of Gaussians fitted on the Hartree-Fock electron densities for rare gases [16]. Our purpose here is to emphasize that such forms Eqs. (33) and (27) are valid for the harmonic oscillator model exactly and are also known in this case in terms of $\rho(x)$ and $\rho^{\prime}(x)$. Another interesting result coming up from the particular case of the harmonic oscillator is that $\xi$ may be formulated only in terms of the kinetic energy densities $t_{\mathrm{TF}}$ and $t_{\mathrm{W}}$. Whether this property is very specific to the present case studied or generalizable to other (three dimensional) systems is an open question at the time of writing.

Finally, one can directly relate the functional derivative $\delta T / \delta \rho$ to the kinetic energy density $t(x)$ and then to the Pauli function in the case of the harmonic oscillator. Indeed, using Eqs. (32) and (17), one readily finds

$\frac{t^{\prime}(x)}{\rho^{\prime}(x)}=\frac{3 t(x)}{\rho(x)}+\frac{\rho^{\prime \prime}(x)}{4 \rho(x)}$,

and then

$$
\frac{\delta T}{\delta \rho(x)}=\frac{3 t(x)}{\rho(x)}+\frac{\rho^{\prime \prime}(x)}{4 \rho(x)}+C(N),
$$

where $C(N)$ is a constant depending on $N$. In the last equation one can recognize in the first term of the right-hand side the homogeneity of the $T F$ functional for one-dimensional electron gas. However the functional does not scale homogeneously because of the $N$-dependent constant $C$. 


\section{Acknowledgements}

The authors gratefully acknowledge financial support by the Flemish Science Foundation (F.W.O.) under the contract G. 0347.97 in which this work has been performed. P.S. would like also to thank the F.W.O. for his postdoctoral position.

\section{References}

[1] R.M. Dreizler, E.K.U. Gross, Density Functional Theory, Springer, Berlin, 1990.

[2] L.H. Thomas, Proc. Camb. Phil. Soc. 23 (1926) 542.
[3] E. Fermi, Z. Phys. 48 (1928) 73.

[4] N.H. March, Adv. Phys. 6 (1957) 1.

[5] N.H. March, Electron Density Theory of Atoms and Molecules, Academic, New York, 1992.

[6] C.F. von Weizsäcker, Z. f. Phys. 96 (1935) 431.

[7] D.A. Kirsnits, Sov. Phys. JETP 5 (1957) 64.

[8] N.H. March, W.H. Young, Nucl. Phys. 12 (1959) 237.

[9] M. Levy, A. Görling, Phil. Mag. B 69 (1994) 763.

[10] C. Herring, Phys. Rev. A 34 (1986) 2614.

[11] C. Herring, M. Chopra, Phys. Rev. A 37 (1988) 31.

[12] G.P. Lawes, N.H. March, J. Chem. Phys. 71 (1979) 1007.

[13] N.H. March, W.H. Young, Proc. Phys. Soc. (London) A 72 (1958) 182

[14] P. Gombas, Phys. Lett. A 28 (1969) 585.

[15] J. Goodisman, Phys. Rev. A 1 (1970) 1574.

[16] B.M. Deb, S.K. Ghosh, Int. J. Quantum Chem. 23 (1983) 1. 